\title{
Optimal Level of Vaginal Fluid Creatinine to Detect Rupture of Amniotic Membranes
}

\author{
Veena Ramasamy ${ }^{1}$, Jaya Vijayaraghavan ${ }^{2}$
}

\begin{abstract}
Aim: To estimate the optimal level of vaginal fluid creatinine to detect rupture of membranes (ROM).

Materials and methods: A total of 300 pregnant women were recruited for the study. Group I consisted of 100 women with a diagnosis of ruptured amniotic membranes confirmed by sterile speculum examination. Group II consisted of 100 women in whom a diagnosis of ROM was suspected but not confirmed by speculum examination. Group III consisted of 100 pregnant women without any complaints taken as a control group. In all 300 women, vaginal fluid washing for creatinine sample was taken. The parameters (age, parity, gestational age at the time of sample collection, amniotic fluid index (AFI), and vaginal fluid creatinine) were compared with one-way analysis of variance (ANOVA) and ScheVe multiple comparison test. Receiver operating characteristic (ROC) curve analysis was used to establish an optimal cut-off concentration. Results: The optimal cut-off value of vaginal fluid creatinine to detect ROM arrived based on ROC curve analysis was $\geq 0.3 \mathrm{mg} / \mathrm{dL}$. The mean vaginal fluid creatinine in group I was $1.097 \mathrm{mg} / \mathrm{dL}$, in group II was $0.3 \mathrm{mg} / \mathrm{dL}$, in group III was $0.068 \mathrm{mg} / \mathrm{dL}$. This study demonstrates that vaginal fluid creatinine could accurately diagnose a ROM with a sensitivity, specificity, positive predictive value, and negative predictive value of $98.36,100,100$, and $97.14 \%$, respectively.

Keywords: Optimal value, ROC curve, Rupture of membranes, Vaginal fluid creatinine.

Journal of South Asian Federation of Obstetrics and Gynaecology (2020): 10.5005/jp-journals-10006-1819
\end{abstract}

\section{INTRODUCTION}

Premature rupture of membranes (ROM) occurs in about $10 \%$ of all pregnancies. Preterm premature rupture of membranes (PPROM) occurs approximately occurring in 3\% of pregnancies, ${ }^{1}$ $0.5 \%$ occurring in pregnancies $<27$ weeks, $1 \%$ of pregnancies $27-34$ weeks, and in $1 \%$ of pregnancies from 34 to 37 weeks. ${ }^{2}$ On the one hand, correct diagnosis of ROM has great importance because the failure of diagnosis can lead to unwanted obstetric complications like chorioamnionitis, preterm birth, and neonatal sepsis. On the other hand, overdiagnosis can lead to unnecessary interventions like hospitalization. Given the controversies surrounding the management of patients with ROM, regardless of the gestational age (GA), it is important to achieve accurate diagnoses by identifying the presence of specific amniotic fluid markers in the vaginal environment. The diagnosis is often based on clinical evaluation, and biochemical markers became a necessity in the case of clinically asymptomatic patients and/or those with unclear premature rupture of membranes (PROM). The current markers available are the measurement of vaginal $\mathrm{pH}$, nitrazine and fern tests, prolactin, alpha-fetoprotein (AFP), di-amine oxidase, insulin-like growth factor binding protein-1 (IGFBP-1), human chorionic gonadotropin, placental alpha microglobulin-1 protein assay (PAMG-1), and fetal fibronectin. Each of these markers has its own advantages and drawbacks, though none of them form a gold standard in the diagnosis of ROM. This study speculates that as fetal urine is the most important source of amniotic fluid in the second half of pregnancy, measurement of creatinine in vaginal fluid for the diagnosis of ROM would be a good reliable indicator as suggested by other studies in recent years. The present study aims at evaluating the reliability of creatinine measurement in the vaginal fluid for the diagnosis of ROM.

\footnotetext{
1,2Department of Obstetrics and Gynecology, Sri Ramachandra Institute of Higher Education and Research, Chennai, Tamil Nadu, India Corresponding Author: Veena Ramasamy, Department of Obstetrics and Gynecology, Sri Ramachandra Institute of Higher Education and Research, Chennai, Tamil Nadu, India, Phone: +91 9940430764, e-mail: veena_392@yahoo.co.in

How to cite this article: Ramasamy V, Vijayaraghavan J. Optimal Level of Vaginal Fluid Creatinine to Detect Rupture of Amniotic Membranes. J South Asian Feder Obst Gynae 2020;12(5):284-287.

Source of support: Nil

Conflict of interest: None
}

\section{Materials and Methods}

This was a prospective observational study conducted at Sri Ramachandra Institute of Higher Education and Research from September 2015 to September 2017. The study included pregnant women with a singleton pregnancy and GA between 26 and 40 weeks of gestation who came for an antenatal visit to Sri Ramachandra Hospital were recruited into the study. The study was approved by the ethical committee of Sri Ramachandra Institute of Higher Education and Research.

The inclusion criteria for the study were antenatal women with singleton pregnancy at $>26$ weeks of gestation, women coming to the hospital with a history of leaking per vaginum, women with clinical findings confirming leak, antenatal women with a history of discharge per vaginum or doubtful leak. Pregnant women with no specific complaints or complications were taken as a control study group. Exclusion criteria for the study: antenatal women who on examination revealed meconium-stained liquor/ 
bloodstained liquor, antenatal women with a sonographically confirmed anomalous fetus, antenatal women in labor with active uterine contraction, antenatal women who have taken vaginal pessaries within 24 hours before the examination, intrauterine fetal demise confirmed by ultrasonography. Women fulfilling the inclusion and exclusion criteria were enrolled in the study. Consent to participate in the study was taken. After a detailed history and general examination of the women who were included in the study, an obstetric examination was done to assess the GA. Presentation of the fetus, fetal heart rate was also assessed.

All women underwent a sterile speculum examination and were divided into three groups. Group I (confirmed group)—women who were confirmed to have ruptured membranes-with a frank leak as evidenced by fluid leaking through cervix/pooling of fluid in the posterior fornix on per speculum examination. In these women, about $3 \mathrm{~mL}$ of the same fluid was aspirated in a $5 \mathrm{~mL}$ syringe and sent to the laboratory for processing to obtain vaginal fluid creatinine value. Group II (suspected group) - women who had a history of leaking per vaginum/discharge per vaginum but per speculum examination did not confirm ROM-i.e., no obvious evidence of leak noted on per speculum examination. In the above-said group of women, on per speculum examination, the cervix was visualized, and $3 \mathrm{~mL}$ sterile water was injected in the posterior fornix and the fluid was aspirated back with the same syringe. The sample obtained was sent to the laboratory. Samples were centrifuged and then processed to obtain creatinine value. Group III (control group): asymptomatic pregnant women served as controls. They had no complaints related to leaking per vaginum/discharge per vaginum. In these women on per speculum examination, the cervix was visualized and $3 \mathrm{~mL}$ of sterile water was injected in the posterior fornix and the same was aspirated back and sent to the laboratory to obtain fluid creatinine value. A baseline amniotic fluid index (AFI) was also done which was followed up the next day in group I and group II, if not already delivered, to confirm if there was a leak. All the women in the group I and group II were then followed up until delivery or for 1 week from the time of sample collection, whichever was the latest. Women in group III were not followed up as they only served as controls to get the baseline value of vaginal fluid creatinine in women with unruptured membranes. Most of the women selected in this group were at or near term. The parameters (age, parity, GA at the time of sample collection, AFI, vaginal fluid creatinine) were compared with one-way analysis of variance (ANOVA) and ScheVe multiple comparison test. Receiver operating characteristic (ROC) curve analysis was used to establish an optimal cut-off concentration. The results were evaluated with a significance level of $p<0.001$.

\section{Results}

The demographic data collected are shown in Table 1. There was a uniform distribution of age and parity across three groups. Mean AFI in group I-9.78, group II-11.2, and group III $-12.8 \mathrm{~cm}$. Incidentally, the occurrence of anemia was seen more in group I and hypothyroid in group II, gestational diabetes mellitus (GDM) in group III. Vaginal

Table 1: Analysis of age, parity, and gestational age in all three groups

\begin{tabular}{llll}
\hline Mean & Group I & Group II & Group III \\
\hline Age & 26 years & 26 years & 28 years \\
Parity & $2(1-3)$ & $2(1-3)$ & $2(1-3)$ \\
Gestational age & $37.6 \pm 3.2$ & $37.5 \pm 2.7$ & $38 \pm 2.4$ \\
AFI & $9.8 \mathrm{~cm}$ & $11.6 \mathrm{~cm}$ & $12.8 \mathrm{~cm}$ \\
\hline
\end{tabular}

fluid creatinine in group I (confirmed group) was of range 0.3-5.1 $\mathrm{mg} / \mathrm{dL}$ with a mean of $1.097 \mathrm{mg} / \mathrm{dL}$. In group I, all 100 observed patients delivered. In group II, vaginal fluid creatinine was of range $<0.1-1.1 \mathrm{mg} / \mathrm{dL}$. With a mean vaginal fluid creatinine of $0.35 \mathrm{mg} / \mathrm{dL}$. In group III, 100 women were observed and none had a significant vaginal fluid creatinine value. This study demonstrates that vaginal fluid creatinine could accurately diagnose a ROM with a sensitivity, specificity, positive predictive value (PPV), and negative predictive value (NPV) of $98.36,100,100$, and $97.14 \%$, respectively.

\section{Discussion}

Correct and timely diagnosis of ROM is essential for optimizing pregnancy outcome, and management is based on accurate diagnosis. No cost-effective, noninvasive diagnostic gold standard test has yet become available. The traditional noninvasive diagnostic methods used include pooling of vaginal fluid on speculum examination, microscopic fern test, $\mathrm{pH}$ examination of cervicovaginal discharge (nitrazine test), have limitations of simplicity, and diagnostic accuracy, especially over time since membrane rupture. Commercially available kits like investigating IGFBP-1 (Actim PROM), PAMG-1 (AmniSure) though form a good diagnostic tool, are not effective due to the cost involved and they also do not have $100 \%$ sensitivity, specificity to form a gold standard of diagnosis. However, the gold standard for diagnosis of ROM, the amnio-dye test, is invasive and carries the risks of infection, abruption.

Of the 300 women observed in this study, 100 women were confirmed to have leaking $\mathrm{p} / \mathrm{v}$ (ruptured membranes) on per speculum examination (group I). Vaginal fluid creatinine samples were obtained from all these women at the time of examination. All these 100 women who were confirmed to have ruptured membranes had a creatinine fluid level of $\geq 0.3 \mathrm{mg} / \mathrm{dL}$, ranging from 0.3 to $5.1 \mathrm{mg} / \mathrm{dL}$, with a mean vaginal fluid creatinine of $1.097 \mathrm{mg} /$ $\mathrm{dL}$. And it was also observed that all these 100 women delivered within 48 hours of obtaining the sample.

In group II (suspected group), 100 women had a history of leaking $p / v$ or discharge $p / v$, but with per speculum examination did not confirm or rule out leaking per vaginum. In these women, a vaginal fluid creatinine sample was obtained the time of admission. Of these, 20 women had vaginal fluid creatinine of $\geq 0.3 \mathrm{mg} / \mathrm{dL}$. All these women delivered within the next 48 hours of sample collection. They developed active leaking per vaginum within 48 hours or there was a significant drop in AFI when it was repeated after 24 hours. In group II (suspected group), 68 women had vaginal fluid creatinine of $<0.3 \mathrm{mg} / \mathrm{dL}$, all these women were observed no further active intervention was done. Pregnancy was continued for a minimum period of 1 week. Table 2 summarizes the outcome of pregnancies in group II women.

In group II, 32 women required termination of pregnancy for the following reasons. Twenty women had vaginal fluid creatinine

Table 2: Outcome observed in group II (suspected group) patients

\begin{tabular}{|c|c|c|c|}
\hline $\begin{array}{l}\text { Number of } \\
\text { patients }\end{array}$ & $\begin{array}{l}\text { Range of vaginal } \\
\text { fluid creatinine }\end{array}$ & $\begin{array}{l}\text { Mean of vaginal } \\
\text { fluid creatinine }\end{array}$ & Outcome \\
\hline \multirow[t]{2}{*}{100} & $<0.1-1.1 \mathrm{mg} / \mathrm{dL}$ & $0.35 \mathrm{mg} / \mathrm{dL}$ & $\begin{array}{l}\text { 68-pregnancy } \\
\text { prolonged for } \\
>1 \text { week }\end{array}$ \\
\hline & & & $\begin{array}{l}\text { 32-pregnancy } \\
\text { terminated (as } \\
\text { explained later) }\end{array}$ \\
\hline
\end{tabular}


of $\geq 0.3 \mathrm{mg} / \mathrm{dL}$ and subsequently leaked or had drop-in AFI and delivered within $24-48$ hours. In the same group in 10 other women with vaginal fluid creatinine of $<0.3 \mathrm{mg} / \mathrm{dL}$, pregnancy had to be terminated because of other obstetric indications. Such as fetal distress, ${ }^{2}$ elective induction ${ }^{8}$ with an indication for inductions being GDM/overt diabetes mellitus, ${ }^{3}$ gestational hypertension, ${ }^{2}$ with a doubtful leak at 40 weeks of gestation, ${ }^{1}$ isolated oligohydramnios with intrauterine growth restriction (IUGR) ${ }^{2}$ not related to ROM at primary obstetrician's discretion. Two women despite creatinine being $<0.3 \mathrm{mg} / \mathrm{dL}$ had a fall in AFI and pregnancy had to be terminated. Hence, the two women could be taken as a falsenegative.

In group III, 100 women were enrolled control. These women were admitted for safe confinement in the hospital with no complaints of discharge per vaginum or leaking per vaginum. Of these 100 women samples taken at the time of per speculum examination revealed that $91 \%$ of women studied had nil creatinine detected in the vaginal fluid, 9 other women had creatinine level of $<0.09 \mathrm{mg} / \mathrm{dL}$, with a mean vaginal fluid creatinine of $0.068 \mathrm{mg} /$ $\mathrm{dL}$. The outcome was not analyzed in this group of women. The range of vaginal fluid creatinine in group III women was from nil to $0.09 \mathrm{mg} / \mathrm{dL}$. There was a significant difference in the mean value of vaginal fluid creatinine across all three groups suggesting that vaginal fluid creatinine is a good reliable indicator in diagnosing ROM with a " $p$ value $<0.001$ ". The optimal vaginal fluid creatinine value was obtained by applying the ROC curve (Fig. 1 and Table 3).

Our study correlated well with the findings of the study conducted by Zanjani and Haghighi who also used vaginal fluid creatinine to detect ROM. Our study also correlated well with a study conducted by El-Sabee et al., Kafali et al., and Li and Chang. Though the slight difference may be attributed to the study design as they analyzed both vaginal fluid urea and creatinine. Li and Chang

Table 3: Mean value of vaginal fluid creatinine in different groups

\begin{tabular}{lllll}
\hline Groups & Group I & Group II & Group III & $\begin{array}{l}\text { Optimal } \\
\text { value }\end{array}$ \\
\hline $\begin{array}{l}\text { Mean } \\
\text { vaginal fluid } \\
\text { creatinine }\end{array}$ & $1.097 \mathrm{mg} / \mathrm{dL}$ & $0.3 \mathrm{mg} / \mathrm{dL}$ & $0.068 \mathrm{mg} / \mathrm{dL}$ & $0.3 \mathrm{mg} / \mathrm{dL}$ \\
\hline
\end{tabular}

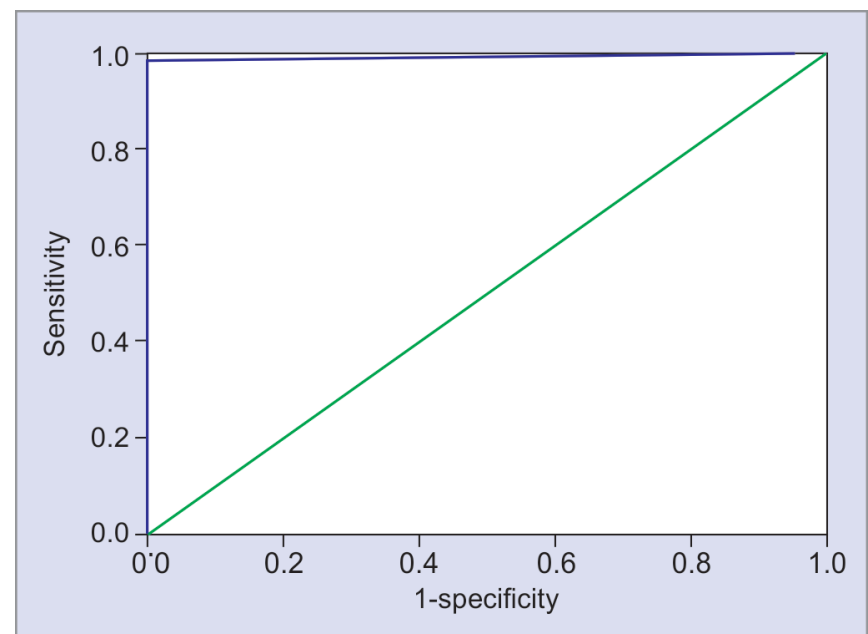

Fig. 1: ROC curve. ROC curve drawn for different cut-off values. The point nearest to the left upper corner was corresponding to mean of $0.3 \mathrm{mg} /$ $\mathrm{dL}$. And this is taken as optimal cut-off value found that measurement of creatinine in vaginal fluid was easier and cheaper than human chorionic gonadotropin (hCG) and AFP. The study group consisted of 54 women in their third trimester of pregnancy with a diagnosis of PROM established by inspection of vaginal pooling and the control group consisted of 34 pregnant women with intact membranes. The results showed a significant difference of $p<0.001$. The sensitivity, specificity, PPV, and NPV were $90,100,100$, and $90.9 \%$, respectively, which was similar to our study. ${ }^{2}$

Kafali et al. reported that determination of urea or creatinine in vaginal fluid for the diagnosis of PROM is a reliable, simple, and rapid test with sensitivity, specificity, NPV, and PPV of $100 \%$. Kafali et al. also speculated that analysis of vaginal creatinine and urea could be used as a fetal maturation test in cases of preterm labor as the creatinine level of amniotic fluid depends on GA. Creatinine values in the amniotic fluid that best represent fetal maturity was 1.5-2 $\mathrm{mg} / \mathrm{dL} .{ }^{3}$ A creatinine concentration of $1.75 \mathrm{mg} / \mathrm{dL}$ or more correlates significantly with a GA of 37 weeks or more. This assessment was, however, not part of our study. El-Sabee et al. studied a total of 96 women who were included in the study, divided into three groups: with 32 women in each group. They had evaluated both urea and creatinine and found the optimal cut-off by ROC curve to be 0.9 $\mathrm{mg} / \mathrm{dL}$ for creatinine and urea to be at $9 \mathrm{mg} / \mathrm{dL}^{4}$

Zanjani and Haghighi reported that the evaluation of vaginal fluid creatinine concentration had $96.5 \%$ sensitivity, $100 \%$ specificity, $100 \%$ PPV, and $96.8 \%$ NPV when a cut-off value of $0.5 \mathrm{mg} /$ $\mathrm{dL}$ was used. They concluded the creatinine level in vaginal fluids a valid and simple test. It costs much less than hospitalization or other tests. Zanjani and Haghighi analyzed the sensitivity, specificity, PPV, and NPV of varying levels of vaginal fluid creatinine. ${ }^{5}$

Lee et al. had done a study on the measurement of placental alpha-microglobulin-1 in cervicovaginal discharge to diagnose a ROM. ${ }^{6}$ They had observed 184 women of GA 11-42 weeks, ROM was diagnosed in $76 \%$ using conventional clinical assessment (nitrazine test) and 88\% using placental alpha-microglobulin-1. Lee et al. had concluded that the placental alpha-microglobulin-1 immunoassay is a rapid and accurate method for confirming the diagnosis of ROM and that its performance appeared to be superior to conventional clinical assessment like pooling, nitrazine, ferning, and the nitrazine test alone. It is noted that the specificity and PPV of vaginal fluid creatinine done in our study was $100 \%$ while that of nitrazine test and AmniSure was $<100 \%$. The sensitivity and NPV were significantly higher in our study than those observed for nitrazine test and AmniSure test. Tejjada et al. used IGFBP-1 to improve the diagnostic accuracy of ROM. ${ }^{7}$ They had also reported the sensitivity, specificity, PPV, and NPV of fern test, AFI $<5 \mathrm{~cm}$, bromothymol, and IGFBP-1. ${ }^{8}$ Our study had comparable specificity and PPV to bromothymol and AFI but had a better sensitivity and NPV compared with the rest.

Gurbuz et al. observed that measurement of vaginal fluid creatinine was an extremely useful marker in the doubtful case of PROM. In these cases, new methods such as AFP, beta hCG, and fetal fibrinonectinin were investigated. However, they were found to have low specificity owing to overlap between the values of hCG, AFP, and fibrinonectinin in patients with or without intact membranes. They concluded that creatinine assay was cheaper and faster than other methods and had higher sensitivity and specificity to establish an accurate diagnosis. They suggested that vaginal fluid creatinine could become a possible candidate to become a gold standard test for PROM. Gurbuz et al. reported that 
the sensitivity, specificity, PPV, and NPV were all 100\% in detecting PROM by evaluation of vaginal fluid creatinine with a cut-off value of $0.12 \mathrm{mg} / \mathrm{dL}$ and sensitivity. ${ }^{9}$

The limitation of the study used only vaginal fluid creatinine assessment based on the clinical findings on speculum examination. We did not use other additional modalities like nitrazine tests to confirm or refute the leak. Chance of false-negative and rejection of certain samples, though very less in our study may have been avoided by the single person collecting the sample which may not be feasible in a true life setting.

\section{Conclusion}

This study demonstrates that vaginal fluid creatinine could accurately diagnose a ROM with a sensitivity, specificity, PPV, and NPV of $98.36,100,100$, and $97.14 \%$, respectively, with the optimal vaginal fluid creatinine to detect leak being $0.3 \mathrm{mg} / \mathrm{dL}$.

\section{References}

1. Mercer BM. Preterm premature rupture of the membranes: current approaches to evaluation and management. Obstet Gynecol Clin North Am 2005;32(3):411. DOI: 10.1016/j.ogc.2005. 03.003.

2. Li HY, Chang TS. Vaginal fluid creatinine, human chorionic gonadotropin and alpha-fetoprotein levels for detecting premature rupture of membranes. Zhonghua Yi Xue Za Zhi (Taipei) 2000;63(9):686-690.

3. Kafali $H$, Oksüzler $C$. Vaginal fluid urea and creatinine in diagnosis of premature rupture of membranes. Arch Gynecol Obstet 2007;275(3):157-160. DOI: 10.1007/s00404-006-0240-1.

4. Kafali $\mathrm{H}, \mathrm{Oksüzler} \mathrm{C}$. Vaginal fluid urea and creatinine as biomarkers of prelabor rupture of membranes (PROM). J Cairo Univ 2015;83(2):173179. www.medicaljournalofcairouniversity.net.

5. Mansooreh S. Vaginal fluid creatinine for the detection of premature rupture of membranes. J Obstet Gynaecol Res 2012;38(3):505-508. DOI: 10.1111/j.1447-0756.2011.01692.x.

6. Lee SE, Park JS, Norwitz ER, et al. Measurement of placental AlphaMicroglobulin-1 in cervicovaginal discharge to diagnose rupture of membranes. Obstet Gynecol 2007;109(3):634-640. DOI: 10.1097/01.AOG.0000252706.46734.0a.

7. Kumar D, Moore RM, Mercer BM, et al. The physiology of fetal membrane weakening and rupture: insights gained from the determination of physical properties revisited. Placenta 2016;42:5973. DOI: 10.1016/j.placenta.2016.03.015.

8. Martinez de Tejada B, Boulvain M, Dumps P, et al. Can we improve the diagnosis of rupture of membranes? the value of insulin-like growth factor binding protein-1. BJOG 2006;113(9):1096-1099. DOI: 10.1111/j.1471-0528.2006.01028.x. www.blackwellpublishing.com/ bjog.

9. Gurbuz A, Karateke A, Kabaca C. Vaginal fluid creatinine in premature rupture of membranes. Int J Gynaecol Obstet 2004;85(3):270-271. DOI: $10.1016 /$ j.ijgo.2003.09.012. 